\title{
Überlegungen zum chemischen Anzünder auf Kalziumoxidbasis
}

\author{
von Ferdinand Nibler
}

\begin{abstract}
Überblick
In der älteren, d.h. vor etwa 1500 entstandenen, kriegstechnischen Literatur ${ }^{1}$ werden verschiedentlich Anzünder beschrieben, die die exotherme Reaktion von Kalziumoxid und Wasser, d.h. das „Löschen von gebranntem Kalk“, als Energiequelle zum Anzünden einer Wirkladung unterschiedlichster Zusammensetzung benutzen. In jüngster Zeit wurde das Thema der selbstentzündlichen Kriegsfeuer wieder aufgegriffen und von Kramer ${ }^{2}$ und Tittmann ${ }^{3}$ kontrovers diskutiert. Hier folgen Überlegungen zur Sache, d.h. es soll der Frage nachgegangen werden, inwieweit solche Anzünder realistisch sind und ob sie mit den Mitteln der Zeit um 1400 machbar waren.
\end{abstract}

\begin{abstract}
The military literature of the $13^{\text {th }}$ to $15^{\text {th }}$ century deals frequently with recipes for igniting devices based on the exothermic reaction of unslaked lime and water. The reaction's energy is used to heat up sulfur to $260^{\circ} \mathrm{C}$. On reaching this temperature sulfur exposed to air starts to burn, acting as a primer for further inflammable materials. Two examples of those recipes are analyzed, partly on a more physical point of view, partly examining the suggested military use. Summing up one might say that some of those igniting devices could work under special conditions, some would be more or less useless under a military point of view. Some recipes seem to be realistic, some phantastic.

The analysis shows the need for experiments because only experimental evidence can give answers to the remaining questions on working conditions and reliability of the abovementioned devices.
\end{abstract}

$$
* * *
$$

1 Übersicht z.B. bei S.J. von Romocki, Geschichte der Explosivstoffe, Bd. 1, Nachdruck der Ausgabe Berlin 1895, Hildesheim 1983, Kapitel I bis VI und bei James R. Partington, A History of Greek Fire and Gunpowder, Nachdruck der Ausgabe Cambridge 1960, Baltimore u. London 1999, Kapitel I u. II.

2 Gerhard W. Kramer, Das Pyr Autómaton - die selbstentzündlichen Feuer des Mittelalters, in: Waffen- und Kostümkunde 44, 2002, S. 49-61.

3 S. Beitrag von Wilfried Tittmann in diesem Heft. 
Die Untersuchung der überlieferten Rezepte zu chemischen Anzündern auf Kalziumoxidbasis beschränkt sich auf den sachlichen Inhalt, dessen Korrektheit ohne kritische Untersuchung der Quelle unterstellt wird. Als willkürlich herausgegriffenes Beispiel folgt hier ein Rezept aus dem Feuerwerkbuch ${ }^{4}$ :

$(229)^{5}$ Wie man macht das sich wasser anzündt.

Wiltu ein matery machen das sich wasser anzind /so nimm vngeleschtenn kalck vnd als vil schwebels an der wag /vnnd auß der matery mach ein dacht/vnd spreng darauff wasser so entzünt es sych /vnd geüst du öl darauff so erlischet es.

In der Übertragung:

(229) Wie man macht, dass sich [etwas] [mit] Wasser entzündet.

Willst du ein Materi[al] machen, das sich [mit] Wasser entzündet, so nimm ungelöschten Kalk und gleichviel Schwefel mit einer Waage. Aus diesem Material mach einen Docht (d.h. „Anzündkörper“) und sprenge Wasser darauf, so entzündet es sich. Und gießt du Öl darauf, so entzündet es sich nicht. ${ }^{6}$

Der Autor dieses Rezeptes meint mit „Docht“ wohl das, was modern „Anzünder" genannt wird. Im weiteren Text wird nur noch der moderne Ausdruck gebraucht werden. Interessant ist der Nachsatz, aus dem die Verblüffung des Autors darüber ersichtlich ist, dass ein Feuer mit dem sprichwörtlich ein Feuer nährenden Öl hier nicht zustande kommt. „Erlischt" wird dabei als Gegenteil von „entzünt es sych“ als „es entzünt sych nicht“ gedeutet, nicht als kaum vorstellbares Löschen eines bereits brennenden Schwefelfeuers mit brennbarem Öl. Oder waren um 1400 schon nicht brennbare oder mindestens schwer entflammbare Öle bekannt?

Da „ein Material“ aus zwei Komponenten gemacht werden soll, deutet das auf eine Mischung der zwei Substanzen Schwefel und Kalk, wobei offen bleibt, ob eine grobe Mischung aus z.B. erbsengroßen Teilchen oder eine feine Mischung aus gepulverten Substanzen gemeint ist. Offen ist auch ob ein Material der ganze Anzünder ist, der dann auch aus z.B. zwei Schichten übereinander oder aus zwei Blöcken nebeneinander bestehen könnte. Eine weitere offene Frage ist, welche Form der Anzünder haben soll oder muss, denn die von dem Wort Docht suggerierte Form „dünn und länglich“ ist, wie die weiteren Überlegungen zeigen werden, nicht unbedingt zwingend, eher irreführend.

4 Z.B. im Druck 1529 bei Heinrich Stainer, als Faksimile hg. von Wilhelm Hassenstein, Das Feuerwerkbuch von 1420, München 1941, S. 21 (neue Zählung Hassenstein).

5 In dem vom Verfasser erarbeiteten Papier Das Feuerwerkbuch in synoptischer Darstellung zweier anonymer Originaltexte - Transkription und Textvergleich des Manuskriptes Ms 362 von 1432 und des sogenannten Feuerwerkbuches von 1420 (gedruckt 1529) mit Anmerkungen zu den Texten hat dieser Abschnitt die laufende Nummer 229. Diese mit vielen Anmerkungen und einer neuhochdeutschen Übertragung versehene synoptische Darstellung kann vom Verfasser bezogen werden über www.feuerwerkbuch.de.

6 Frei aus ,so erlischt es“. 
Ein zweites, ebenso willkürlich herausgegriffenes Beispiel, ist das folgende Rezept aus dem Bellifortis ${ }^{7}$ von Conrad Kyeser:

Ut a pluvia accendatur domus: Recipe uncias duas recipe sulfuris et uncias calcis vive tres et sextam partem et aliquantulum de petroleo et de istis omnibus fac confeccionem et pone supra tectum et pluvia adveniente ardebit. De eadem confeccione fac ymaginem de qua videbis effectum si sub aqua posueris ardebit donec aceto vel urina extinguatur.

In der Übertragung (nach Quarg):

Damit durch Regen ein Haus angezündet werden kann: Nimm 2 Unzen Schwefel und 3 Unzen ungelöschten Kalk und etwas mehr als den sechsten Teil ${ }^{8}$ [Petroleum] $]^{9}$, und von dem Allen mache eine Mischung und lege sie auf das Dach, und bei einsetzendem Regen wird es brennen. Von derselben Mischung mache ein [Gebilde] ${ }^{10}$, von dem Du die Wirkung sehen wirst, wenn Du es unter Wasser hältst: Es wird brennen, bis es mit Essig oder Urin gelöscht wird.

Dieses zweite Rezept unterscheidet sich vom ersten in einem wesentlichen Punkt: Der Anzünder enthält neben dem eigentlichen Reaktionsmaterial, nämlich Kalziumoxid und Schwefel, auch noch Petroleum als Brandsubstanz zur Verbesserung der Wirkung. Bemerkenswert sind auch die absoluten Mengenangaben in Unzen (zu etwa $30 \mathrm{~g}$ ), weil damit ein Anhalt für die Größe derartiger Anzünder gegeben ist. Im vorliegenden Fall beispielsweise hätte der gesamte Anzünder einschließlich der Brandsubstanz eine Masse von knapp $200 \mathrm{~g}$ bzw. ein Volumen von etwa $100 \mathrm{~cm}^{3}$, entsprechend einem Würfel von etwa 4,6 cm Kantenlänge.

Beide Rezepte gehören zu der Gruppe „Anzünder ohne Sauerstoffträger (=Salpeter)“. Die ebenfalls überlieferten Rezepte der Gruppe „Anzünder mit Sauerstoffträger", die wahrscheinlich jüngeren Datums sind, werden hier nicht betrachtet.

Die Grundlage des chemischen Anzünders ist die exotherme Reaktion von Kalziumoxid $\mathrm{CaO}$ (gebrannter Kalk) mit Wasser:

$$
\mathrm{CaO}+\mathrm{H}_{2} \mathrm{O} \rightarrow \mathrm{Ca}(\mathrm{OH})_{2}+\mathrm{W}
$$

Die bei dieser Reaktion, dem Löschen von Kalk, freiwerdende Energie W interessiert hier besonders, weil damit der eigentliche Anzündvorgang initialisiert wird. W hängt von der reagierenden Menge an Kalk und Wasser

7 Conrad Kyeser, Bellifortis, in der Faksimileausgabe mit Umschrift und Übersetzung von Götz Quarg, Düsseldorf 1967, fol 102 ${ }^{\mathrm{r}}$ bzw. S. 73.

8 Also „etwas mehr als den sechsten Teil von 5 Unzen“, was als sechsten Teil der Gesamtmasse „etwa eine Unze Petroleum“ ergibt.

9 Quarg übersetzt ohne Erklärung und deshalb nicht nachvollziehbar „petroleum“ mit „Ziegelsteinöl“.

10 Quarg übersetzt „ymago“ mit „Bildnis“. 
ab. Reagieren die Molgewichte von $\mathrm{CaO}, 56 \mathrm{~g}$, und $\mathrm{H}_{2} \mathrm{O}, 18 \mathrm{~g}$, so ist $\mathrm{W}=63$ $\mathrm{kWs}$ oder $\mathrm{W}=63 \mathrm{~kJ} .{ }^{11}$ Um diese Energie besser beurteilen zu können, wird die auf das Einheitsvolumen $1 \mathrm{~cm}^{3}$ reagierender Massen bezogene Energie $\mathrm{W}_{\mathrm{v}}$ eingeführt. Die Dichte von $\mathrm{Ca}(\mathrm{OH})_{2}$ ist etwa 2 bis $2,3 \mathrm{~g} / \mathrm{cm}^{3}$, woraus sich das Volumen des Reaktionsproduktes ergibt zu

$$
V=\frac{56+18}{2.1}=35.2 \mathrm{~cm}^{3} .
$$

Daraus folgt weiter die Energie pro Einheitsvolumen $\mathrm{W}_{\mathrm{v}}=1,79 \mathrm{kWs} / \mathrm{cm}^{3}$. Die Leistung während des Ablaufs der Reaktion ist entsprechend $P_{\mathrm{V}}=1,79 \mathrm{~kW} / \mathrm{cm}^{3}$. Die bei dieser Reaktion umgesetzte Energie ist also beträchtlich. Was bewirkt nun diese Energie? Zunächst einmal erwärmt sich das Reaktionsprodukt selbst auf eine Temperatur, die über der als Ausgangstemperatur zu betrachtenden Umgebungstemperatur liegt. Sobald die Temperatur des aktiven Volumens aber über der Umgebungstemperatur liegt, wird Energie an die Umgebung abgegeben und diese dadurch erwärmt. Die unmittelbare Umgebung wird dabei zuerst und am stärksten erwärmt, die weitere Umgebung später - es entsteht ein sich im Laufe der Zeit abbauendes Temperaturgefälle bis die Umgebung dieselbe Temperatur wie das aktive Volumen hat, eine Mischtemperatur zwischen der hohen Ausgangstemperatur des aktiven Volumens und der ursprünglichen Umgebungstemperatur. Betrachten wir jetzt nur die Anfangsphase dieses Ausgleichsvorganges und nur die unmittelbare Umgebung, d.h. ein relativ kleines Volumen in unmittelbarem Kontakt mit dem aktiven Volumen. Nehmen wir an, dass das Umgebungsvolumen von Wasser erfüllt sei und nehmen wir weiter an, dass der Energieübergang nur über die sechs Begrenzungsflächen des als Würfel gedachten aktiven Volumens erfolgt und dass der Bereich der „unmittelbaren Umgebung" aus sechs plattenförmigen Teilvolumina besteht. Setzen wir die Plattenhöhe - willkürlich, aber sicher zulässig - mit $1 / 6 \mathrm{~cm}$ an, so ergibt sich als das zu erwärmende Volumen der „unmittelbaren Umgebung“ ebenfalls $1 \mathrm{~cm}^{3}$, erfüllt von Wasser. Damit haben wir die konkrete Frage, auf welche Temperatur dieses Umgebungswasser erwärmt wird.

Wir haben also $1 \mathrm{~cm}^{3}$ aktives Material - Kalziumhydroxid - und fragen nach der Erwärmung von $1 \mathrm{~cm}^{3}$ Wasser. Da das aktive Volumen nur solange Energie abgibt, bis die Umgebung und das aktive Volumen dieselbe Temperatur haben, verbleibt ein Teil der Gesamtenergie im aktiven Volumen. Die Temperatur $\mathrm{T}$ im aktiven Volumen und in der unmittelbaren Umgebung ergibt sich also als eine Funktion des Ortes $\mathrm{x}$ und der Zeit $\mathrm{t}$ : $\mathrm{T}(\mathrm{x}, \mathrm{t})$. In der Abbildung sind drei zeitlich aufeinanderfolgende Temperaturverläufe $\mathrm{T}(\mathrm{x}, \mathrm{t})$ stark schematisiert qualitativ (und darum ohne Maßstab!) dargestellt.

11 Zu den Einheiten: Die Einheit Kalorie cal, auch Kilokalorie kcal (Große Kalorie genannt), ist veraltet. Sie wurde abgelöst von der Wattsekunde Ws, auch Joule J genannt. Für die Umrechnung gilt $1 \mathrm{Ws}=0,239 \mathrm{cal}$ bzw. $1 \mathrm{cal}=4,1841 \mathrm{Ws}$. In älteren Darstellungen ist deshalb $\mathrm{W}=15 \mathrm{kcal} z u$ finden. 


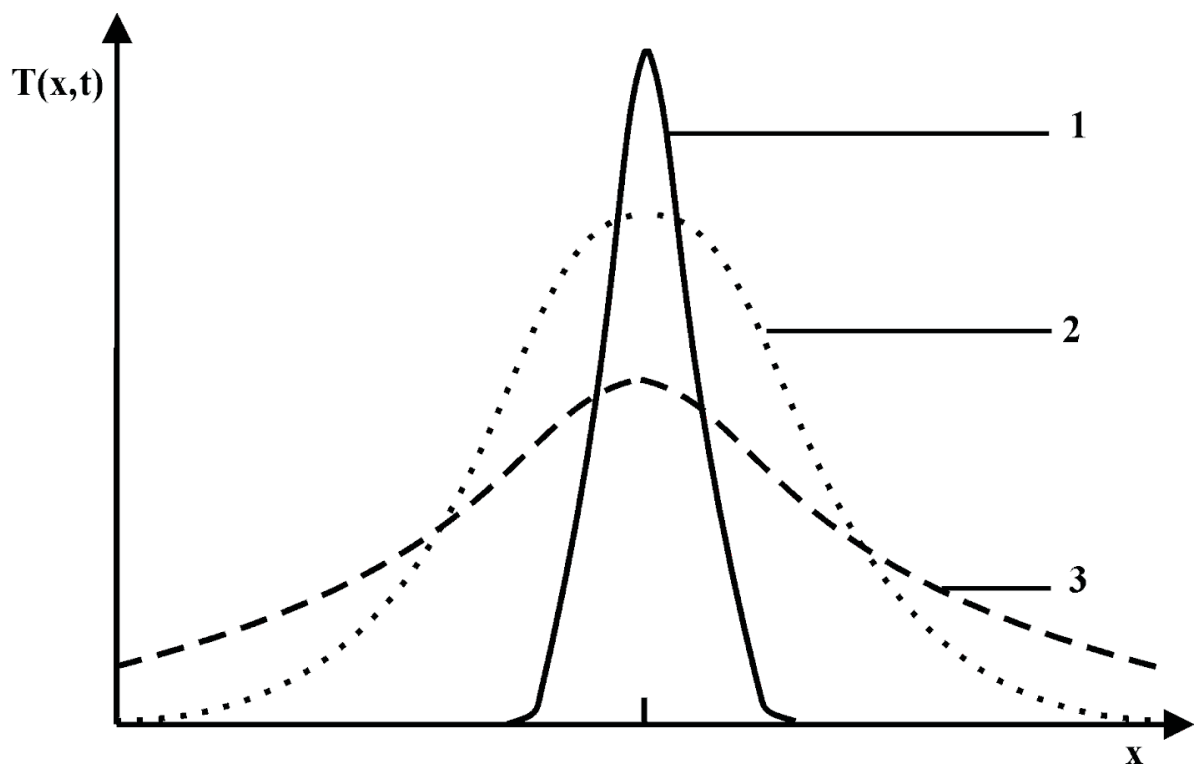

Abb. 1: Temperaturverlauf $\mathrm{T}(\mathrm{x}, \mathrm{t})$ im aktiven Volumen und der unmittelbaren Umgebung, qualitativ und schematisch - Kurve 1 ,am Ende der Reaktion“, Kurve 2 „kurz danach“, Kurve 3 „später“.

Als wichtigste Frage stellt sich jetzt die nach der Maximaltemperatur im aktiven Kern am Ende der Reaktion. Zur Erwärmung des Kernes stehen 1,79 kWs zur Verfügung. Damit könnte sich der Kern, ausgehend von $20^{\circ} \mathrm{C}$, auf 400 bis $450^{\circ} \mathrm{C}$ erwärmen und - vgl. Kurve 2 - die , unmittelbare Umgebung““ könnte nach kurzer Zeit bis zu $300^{\circ} \mathrm{C}$ erreichen. Diese Abschätzung gilt aber nur, wenn keine Wasserreste im Kern oder in der unmittelbaren Umgebung verbleiben! Das Restwasser wird nämlich nur bis $100^{\circ} \mathrm{C}$ aufgeheizt, d.h. es begrenzt den weiteren Temperaturanstieg bis es restlos verdampft ist. Erst danach könnte die Temperatur weiter steigen, wenn noch genügend Energie vorhanden ist, da zur Verdampfung ein nicht unbeträchtlicher Teil der Energie verbraucht wird, nämlich ca. $0,33 \mathrm{kWs}$ zur Erwärmung auf $100^{\circ} \mathrm{C}$ und weitere 2,251 kWs zur Verdampfung von $1 \mathrm{~cm}^{3}$ Umgebungswasser ohne Berücksichtigung des Restwassers im Kern. Da insgesamt nur $1,79 \mathrm{kWs} / \mathrm{cm}^{3}$ zur Verfügung stehen, heißt das, dass $1 \mathrm{~cm}^{3}$ aktiver Kern und $1 \mathrm{~cm}^{3}$ Umgebungs- und Restwasser erstens nicht mehr als $100^{\circ} \mathrm{C}$ erreichen können und zweitens nicht völlig abtrocknen können.

Wenn also zur Initialisierung des Anzünders das Wasser unkontrolliert geschüttet $^{12}$ wird $^{13}$, ist die angestrebte Funktion, nämlich Selbstentzündung des

12 „Geschüttet“ wird hier bewusst gewählt, weil z.B. im Feuerwerkbuch clm 30150 an der korrespondierenden Stelle (Abschnitt 203) „beschüt wirt“ steht.

13 Wenn Jemand den Anzünder mit Wasser beschüttet (vgl. Anm. 12), drängt sich sofort die 
Schwefels an der Luft bei Erreichen des Flammpunktes bei $260^{\circ} \mathrm{C}$, mit Sicherheit nicht möglich. Auch wenn Kalziumoxid und Schwefel im Verhältnis 1:1 gemischt vorliegen, ändert sich wenig. Die freiwerdende Energie pro $\mathrm{cm}^{3}$ halbiert sich, aber auch wenn im aktiven Kern kein oder fast kein Restwasser zu verdampfen ist und damit für den Schwefel die kritische Temperatur von $260^{\circ} \mathrm{C}$ erreichbar sein dürfte, eine Selbstentzündung an der Luft ist wiederum erst möglich, wenn auch das Wasser , in der unmittelbaren Umgebung" verdampft ist. Dieses Umgebungswasser schirmt bis zu seiner Verdampfung den Schwefel nicht nur ab, es begrenzt auch an der äußeren Oberfläche die Temperatur der äußeren Schwefelteile auf $100^{\circ} \mathrm{C}$.

Deutlich besser ist die Situation, wenn „die Materie mit Wasser besprengt wird" wie im ersten Rezept steht oder, modern formuliert, wenn das Wasser dosiert zugeführt wird, ${ }^{14}$ also etwa 0,4 bis $0,5 \mathrm{~g}$ pro $1 \mathrm{~cm}^{3}$ Reaktionsmasse. In diesem und nur in diesem Fall reagieren Kalziumoxid und Wasser zu 100 Prozent und ohne Restwasser. In diesem Fall kann die Temperatur im aktiven Kern auf über $400^{\circ} \mathrm{C}$ ansteigen und der Schwefel in unmittelbarer Umgebung erreicht nach kurzer Zeit die kritische Temperatur von $260^{\circ} \mathrm{C}$ - es kommt zur Selbstentzündung, der Anzünder funktioniert. Die Kurven 1 und 2 stellen das qualitativ dar: Kurve 1 am Ende der Reaktion, Kurve 2 „kurz darauf“ - die Erwärmung der „unmittelbaren Umgebung“ ist deutlich erkennbar.

Betrachten wir jetzt einen - der Einfachheit halber halbkugelförmigen größeren Klumpen Anzündmasse mit einem Volumen von z.B. $1000 \mathrm{~cm}^{3}=11$. Diese Halbkugel hat einen Radius von 7,18 cm. Wenn sie von einer Wasserschicht der Dicke $1 \mathrm{~cm}$ umgeben ist, hat dieses Wasser ein Volumen von rund $450 \mathrm{~cm}^{3}$. Zur Erhitzung auf $100^{\circ} \mathrm{C}$ und anschließenden Verdampfung werden $450 \times 2,581=1161,45 \mathrm{kWs}$ benötigt. Bei der Gesamtenergie von $1790 \mathrm{kWs}$ nach vollständiger Reaktion von $1000 \mathrm{~cm}^{3}$ Anzündmasse könnte dieser knappe halbe Liter Wasser also verdampft werden und auch noch die Flammtemperatur von Schwefel $\left(260^{\circ} \mathrm{C}\right)$ mit der Restenergie von 628,55 kWs zumindest in Teilen des aktiven Kerns erreicht werden, wenn die Reaktion auch in der Tiefe rasch genug abläuft. Die Geschwindigkeit der Reaktion und die Geschwindigkeit ihrer Ausbreitung sind aber nicht bekannt! Aufheizung und gleichzeitige Abkühlung durch Energieabgabe an die Umgebung hängen aber entscheidend von eben diesen beiden Geschwindigkeiten $a b$, weswegen die obige Rechnung zwar zeigt, dass mit einem größeren aktiven Volumen der chemische Anzünder grundsätzlich funktionieren kann, aber nicht zeigt, dass er funktionieren wird! Eine experimentelle Untersuchung erscheint somit zwingend notwendig.

Frage auf, warum wird dann nicht konventionell angezündet? Vermutlich ist dieses „,beschütten" also nicht wörtlich zu interpretieren.

14 Dazu muss aber doch ein ,richtig dosierender“ Mann anwesend sein und es ergibt sich wieder die Frage der Anm. 13! 
Allerdings ist die Annahme einer Halbkugel, die von einer Wasserschicht bedeckt wird, unrealistisch, da das Wasser abläuft und damit die Kuppe des oberen Kugelbereichs freigibt. Zumindest in diesem Bereich, wo die Verdampfung keine entscheidende Rolle spielen dürfte, wird also die kritische Temperatur von $260^{\circ} \mathrm{C}$ erreicht werden können und der Schwefel im Kuppenbereich könnte sich entzünden. Wie kann dieses Feuer die Dachschindeln am Fuß der Halbkugel, die sozusagen ,im Wasser stehen“, entzünden? Auch die Übertragungsladung aus Petroleum o.ä. könnte erst nach dem Abtrocknen der Schindeln wirken. Dieses lässt sich nur experimentell nachweisen.

Dabei ergibt sich ein weiteres Problem: Zur Selbstentzündung von Schwefel ist grundsätzlich Luftsauerstoff von Nöten, dieser dürfte aber eigentlich im Inneren des Reaktionsvolumens nicht zur Verfügung stehen. Und auch die vorgeschlagenen Übertragungsladungen (hier im zweiten Rezept Petroleum, in anderen Rezepten werden Harz, Wachs usw. genannt) sind problematisch, denn sie schirmen zum einen das $\mathrm{CaO}$ vom Wasser und zum anderen den Schwefel vom Luftsauerstoff ab. Wie wirken sich also diese Beimengungen aus, insbesondere bei inniger Vermischung der drei Substanzen? Auch dies kann nur experimentell untersucht werden.

Wenn man den Anzünder durch Regen oder Tau initialisieren möchte, so ist Regen der unkontrollierten Schüttung gleichzusetzen, d.h. nur ein kleiner Schauer mit der etwa richtigen Wassermenge bringt den Anzünder mehr oder minder zufällig vielleicht manchmal zur Funktion. Die Initialisierung durch Tau erscheint grundsätzlich möglich, aber nicht unproblematisch. Bei den in der gemäßigten Zone ${ }^{15}$ durchschnittlichen Taumengen von etwa 0,1 bis $0,3 \mathrm{~mm}$ Tau in einer Taunacht, d.h. 0,01 bis $0,03 \mathrm{~g} / \mathrm{cm}^{2}$ Wasser, kann ein Anzünder mit einer Schichtdicke von $1 \mathrm{~cm}$ nicht ausreichend intensiv reagieren, es würden dazu an die $5 \mathrm{~mm}$ Tau entsprechend $0,5 \mathrm{~g} / \mathrm{cm}^{2}$ Wasser benötigt. Um den Anzünder im Aufbau an die Taumenge anzupassen, könnte man jetzt die Schichtdicke auf etwa $1 \mathrm{~mm}$ reduzieren und damit die vollständige Reaktion ziemlich wahrscheinlich erreichen. Wenn diese dünne Schicht beim Aufbau des Anzünders verwendet wird, ist allerdings mit Problemen beim Wärmeübergang an die Umgebung zu rechnen, da jetzt die Oberfläche in ungünstiger Relation zum Volumen steht - es besteht die Gefahr, dass zu viel Energie verloren geht. Eine kleinkörnige Vermischung ${ }^{16}$ von Schwefel und Kalziumoxid könnte hier aber Abhilfe schaffen. Ein Punkt bleibt allerdings noch offen: Die Reaktion beginnt mit dem Beginn der Tauabscheidung und endet mit dem Ende der Tauabscheidung. Die Zeit dafür ist nicht genau bekannt, aber da sie größenordnungsmäßig etwa eine Stunde ist und somit groß im Verhältnis zur Reaktionszeit, wird die Energie im aktiven Volumen so langsam freigesetzt, dass mit einer Übertemperatur gegenüber der unmittelbaren

15 In den Tropen und Subtropen bis über $3 \mathrm{~mm}$.

16 Das entspricht der Anweisung in Hs. 362, Abschnitt 203 (Abschnittszählung nach Anm. 5) ,und puluer das vnder ain annder“". 
Umgebung von mindestens $260^{\circ} \mathrm{C}$ wegen der sofort beginnenden Wärmeübertragung an die weitere Umgebung nicht gerechnet werden kann. ${ }^{17} \mathrm{Die}$ Kurve 3 in der Abbildung entspricht in etwa diesem Fall. Der Anzünder mit Betauung zur Initialisierung ist somit nach dieser Überlegung nicht funktionsfähig.

Reine Spekulation ist die Frage nach dem weiteren Aufbau. Dass der brennende Schwefel die Balken einer Kriegsmaschine (Blide, Katze o.ä.) direkt entflammt, erscheint eher unwahrscheinlich. Hier könnte man an eine Übertragungsladung auf Basis von Petroleum, Harz oder Terpentinöl denken - dieses wird im Großteil der Rezepte nicht erwähnt.

Damit stellt sich die Frage, wie verlässlich die Angaben in den verschiedenen Rezepten sind. Zum einen gibt es Rezepte wie z.B. das aus dem Feuerwerkbuch entnommene, die zu wenig Information enthalten und somit nur über Experimente geprüft werden können, andere, wie z.B. das aus Bellifortis entnommene, enthalten zumindest in Teilen eindeutige Falschinformationen. Das aus Kalziumoxid, Schwefel und Petroleum angefertigte Gebilde - ohne Sauerstoffträger! - kann unter Wasser nicht brennen. Die nächste Frage, wie man unter Wasser mit Essig oder Urin ein Feuer löschen kann, erübrigt sich damit.

In Anbetracht der Tatsache, dass die überlieferten Rezepte nur im ersten Moment präzise erscheinen, bei genauerem Betrachten aber wenig Information liefern, liegt die Vermutung nahe, dass es sich bei den Rezepten um „Gedankenspiele oder Laborversuche" handelt, die nie im Kriegseinsatz realisiert wurden. Was hätte man im Kriegseinsatz auch damit erreichen können? Sabotage an feindlichen Kriegsmaschinen - warum nicht durch den Einsatztrupp die Maschinen gleich zerstören lassen, statt auf den Tau mit allen Imponderabilien zu warten? Oder wurde der Anzünder nur als jederzeit zur Verfügung stehende „Feuerquelle im Feld“ benutzt und dabei das Wasser genau dosiert? Diese Art der Verwendung erscheint - noch ohne Überprüfung von weiteren Quellen - zwar im ersten Moment spekulativ, aber durchaus möglich und somit vielleicht sogar wahrscheinlich. Vielleicht handelt es sich aber auch um mündlich tradierte Informationen, die der nicht fachkundige Schreiber ungeprüft übernommen hat? Wenn auch hier nur zwei Rezepte zitiert wurden, so lässt sich doch verallgemeinernd feststellen: Es gibt sachlich fundierte Rezepte, die wahrscheinlich oder möglicherweise funktionsfähige Anzünder beschreiben (z.B. Feuerwerkbuch), und fantastische Rezepte (z.B. Bellifortis), die wohl kaum funktionsfähige Anzünder beschreiben.

Vor einer endgültigen oder zumindest fundierten Aussage über die chemischen Anzünder auf Kalziumoxidbasis müssen aber auf jeden Fall prakti-

17 Das dürfte aber immer der Fall sein, denn nach persönlicher Auskunft von Herrn Miller, Deutscher Wetterdienst München, ist die Tauzeit in allen Klimaten auf „,bis zu eine Stunde“ anzusetzen und ist ein ,schlagartiges Ausfallen des Taus“ ausgeschlossen. 
sche Experimente durchgeführt werden, nur textkritische Untersuchungen oder grundsätzliche Betrachtungen und Abschätzungen wie oben reichen nicht aus.

Anschrift des Verfassers: Prof. Dr. Ferdinand Nibler, Robert-Koch-Str. 13, D-85521 Ottobrunn. Email: Dr.F.Nibler@t-online.de. 
